\title{
L'élévation spirituelle à travers la matérialisation du corps féminin dans l'art moderniste catalan
}

Une imagination médiatisée?

\section{Eliseo Trenc}

\section{OpenEdition}

Journals

Édition électronique

URL : http://journals.openedition.org/agedor/2421

DOI : $10.4000 /$ agedor.2421

ISSN : 2104-3353

Éditeur

Laboratoire LISAA

Référence électronique

Eliseo Trenc, «L'élévation spirituelle à travers la matérialisation du corps féminin dans l'art moderniste catalan », L'Ấge d'or [En ligne], 3 | 2010, mis en ligne le 15 février 2010, consulté le 19 avril 2019. URL :

http://journals.openedition.org/agedor/2421; DOI : 10.4000/agedor.2421

Ce document a été généré automatiquement le 19 avril 2019.

L'Âge d'or. Images dans le monde ibérique et ibéricoaméricain 


\section{L'élévation spirituelle à travers la matérialisation du corps féminin dans l'art moderniste catalan}

Une imagination médiatisée?

\section{Eliseo Trenc}

1 Dans ce travail, forcément court, je vais me concentrer sur la production graphique de l'artiste Alexandre de Riquer, essentiellement des illustrations d'ouvrages, des ex-libris et des affiches, mais je pense que certaines de mes analyses pourraient s'étendre au symbolisme plastique moderniste catalan en général (peinture et sculpture). Le problème de la symbolique du corps humain, féminin ou masculin, est une constante dans l'histoire de l'art, et il est connu sous le nom d'allégorie. Mais dans l'allégorie classique, très codifiée, le corps féminin représente toujours des notions abstraites, immatérielles, des vertus, la charité, la bonté, des valeurs morales, la justice, l'égalité, la fraternité, etc. Aussi la seule originalité et implication de l'artiste réside dans le traitement formel d'un thème qui lui, est conventionnel et déterminé par une iconographie figée jusqu'à la fin du XVIII siècle.

2 Lorsque, avec le Symbolisme, le corps féminin va être utilisé par les artistes comme une matérialisation de la vie intime de l'âme du créateur, et de son mystère, il va s'éloigner de la relation claire et univoque entre la représentation iconographique et sa symbolique qui caractérise l'allégorie classique, pour entrer dans une relation plus complexe et plus personnelle, dans laquelle comme le dit Alexandre de Riquer lui-même : «Tout ce qui vit à l'intérieur de soi va essayer de s'extérioriser ». Mais, comme je vais tenter de le montrer, même pour essayer de dévoiler son monde intérieur, l'artiste va puiser obligatoirement dans un répertoire d'images préexistantes, mémorisées et réutilisées donc.

Dans un article sur les sources des affiches d'Alexandre de Riquer, Rafael Cornudella i Carré ${ }^{1}$ montre les emprunts très précis de modèles féminins anglais préraphaélites par l'artiste catalan qui réutilise une figure en la changeant de contextualisation. Ce qui est curieux, par rapport à ce que nous allons voir ensuite, c'est que ces premiers emprunts dés 
piritualisent plutôt les modèles en les rendant plus conventionnels ou prosaïques. Par exemple le personnage féminin visiblement inspiré du haut-relief du sculpteur britannique George Frampton, intitulée Vision, et qui semble une recréation mystique de l'iconographie musicale de Saint Cécile, devient, dans l'affiche de Riquer pour la III Exposition des Beaux-Arts et Industries Artistiques de Barcelone de 1896, une simple allégorie de l'art, les touches de l'orgue de la sculpture étant remplacées par la palette et une petite sculpture que le personnage tient dans ses mains, alors que sa compagne, elle, symbolise l'industrie artistique. De même, la figure féminine, plongée dans la lecture d'un livre illustrant un petit ex-libris de la dessinatrice Alice B. Woodward, est reprise en grand format pour une affiche commerciale de la Granja Avícola de San Luis, dans laquelle le livre qui peut représenter le rêve, l'au-delà de la réalité, l'évasion esthétique, est remplacé par les poussins de l'élevage avicole. Une première conclusion que l'on peut tirer de ces exemples est que le personnage féminin en soi n'a pas une signification particulière, et que c'est l'association avec un contexte, des attributs qui va lui donner son sens. Pour Alexandre de Riquer, à la fois artiste et poète, je m'appuierai sur les textes qui accompagnent souvent les images, pour les interpréter.

4 Je vais m'intéresser d'abord à une figure féminine récurrente dans l'œuvre de Riquer qui, par son attitude même, exprime une idée d'élévation, de désir d'envol. Il s'agit d'une femme qui détache sa silhouette vue de profil, plus ou moins dénudée, la tête renversée en arrière et de laquelle retombe une abondante chevelure très Art Nouveau, et qui lève les bras, tenant des objets différents suivant les versions. La première version de cette iconographie apparaît en 1887 dans une série de vingt plafonds décoratifs, des détrempes sur toile, destinés à décorer l'appartement d'un dentiste de Barcelone, Bofill. Ces peintures décoratives, qui imitent des tapisseries avec le motif d'un grand encadrement floral sur lequel se détache le motif iconographique, sont considérées comme un des premiers indices en Catalogne du japonisme et du culte à la nature, une nature stylisée. Certains des personnages féminins sont des allégories de la médecine, la chirurgie, la chimie, mais d'autres n'ont pas de caractère symbolique déterminé, elles représentent des nymphes, des figures féminines qui sont comme des émanations de la beauté de la nature. Celle qui nous intéresse a pour titre, Figure féminine sentant des pavots, et nous y voyons une figure féminine ailée, comme une sylphide, vêtue jusqu'à la taille d'une sorte de longue jupe médiévale, qui se renverse en arrière, mettant en valeur sa poitrine nue, les bras levés au-dessus de sa tête, et sentant un pavot qu'elle tient dans sa main, comme si elle était déjà emportée dans un autre univers par l'odeur du pavot, dont, je le rappelle, est tiré l'opium. L'idée de transport, d'évasion, est donc déjà présente dans cette image, mais sans transcendance.

5 Une autre variante de ce motif, avec des modifications sur lesquelles je vais revenir, apparaît dans la page de titre du numéro exceptionnel de janvier 1897 de la revue barcelonaise La Ilustración Artística, consacré à une édition illustrée par Riquer de Rinconet e y Cortadillo de Cervantes. La principale modification vient du fait que la figure féminine est agenouillée, ce qui est un signe de révérence et d'hommage, que sa tête est moins renversée en arrière et qu'au bout de ses bras dressés, ses mains tiennent une plume d'oie, avec laquelle elle semble écrire le nom de l'écrivain, et une couronne de lauriers. Malgré le caractère Art Nouveau de l'image, la sensualité due à la longue chevelure bouclée de la femme, à son corps à demi dénudé, vêtu de voiles légers, et le décor floral en bas à gauche de la composition, il s'agit bien évidemment d'une allégorie de la gloire qui 
couronne le génie de Cervantes, une image explicite sans connotations transcendantes ici non plus.

6 À la même époque, on retrouve un personnage féminin dans la même position que celui de la peinture décorative de 1887, illustrant la couverture du numéro 9 de décembre 1898 de la revue Luz, composition à nouveau utilisée, mais en format plus petit et en couleur violette et jaune, comme illustration d'un des poèmes en prose du recueil poétique Crisant emes du peintre-poète catalan publié en 1899. Curieusement, ce poème (p. 19-22) est consacré à une abeille qui, au lieu de travailler comme une ouvrière exemplaire à la ruche, va préférer se perdre dans la contemplation de la nature la plus sauvage, au bord d'un étang bleu où dorment les traditions et le mystère de l'existence. On a, p. 19, une première illustration stylisée mais réaliste de cette abeille qui s'approche d'une branche de lys qu'elle va butiner. Mais, au milieu du poème, la voix poétique nous dit que cette abeille, enfermée dans le calice d'une fleur qui lui sert de palais, y a vécu la vie de l'âme, et on comprend donc qu'elle s'est transformée, qu'elle est devenue la voix poétique, elle aussi étrangère au monde du travail et livrée à la contemplation et au pouvoir de la création qui lui ouvre une autre vie, une sorte de paradis décrit dans le texte du poème comme un ciel plus vaste, peuplé d'êtres immortels. C'est pour cette raison que, dans la deuxième illustration du poème, l'abeille a disparu, remplacée par une sorte de nymphe dont seul le buste émerge du près duquel elle semble s'élever, tout en en étant une sorte d'émanation. On retrouve la vision de profil, la poitrine nue, le bras levé, la tête renversée, mais ici la nymphe a une longue chevelure bouclée, en arabesques, typique de l'Art Nouveau, très sensuelle, et son regard semble se perdre dans le ciel de la composition où brille le disque de la lune. Ce personnage féminin est moins décoratif, plus stylisé, plus spirituel avec le chromatisme violet de l'illustration et la verticalité à la fois de la composition et des épis et des joncs, que celui de la peinture de 1887, et au lieu d'être inscrit dans un cadre floral purement décoratif, il est immergé dans une nature exubérante qui finira par l'engloutir, car l'abeille - âme du poète - va s'endormir dans le calice du lys qui va se fermer avec la nuit, au milieu des visions et des enchantements chromatiques du crépuscule. Il y a une sorte de fusion entre l'âme de la voix poétique et la nature dans le poème, avec la métaphore de l'abeille qui va rester à jamais prisonnière dans la corolle du lys, elle va y dormir le sommeil de la mort, pendant que les voix de la nature semblent entonner un hymne à la lune, symbole à la fois de mort et de résurrection. C'est pour cela peut-être que Riquer nous présente son personnage féminin, comme hypnotisé par la clarté de la lune, comme une nymphe qui rend hommage par son chant mélancolique à l'astre de la nuit. Le monde poétique que crée Riquer s'intériorise au fur et à mesure qu'il réélabore ses expériences du monde sensible, ce que l'anglais traduit par un évocateur jeu de mots, le « landscape » devient « inscape », un monde dans lequel la description des éléments naturels prend un aspect spirituel, ce qui convertit la nature en un monde idyllique, paradisiaque. Il y a donc une fusion dans le personnage féminin entre l'âme de la voix poétique et la nature, sous la forme de cette nymphe qui, d'une part, s'envole vers le monde des visions, de la fantaisie et du rêve, mais, d'autre part, semble faire partie de la nature, en être une émanation. Elle ne peut donc pas s'en détacher et, comme l'abeille, en est prisonnière à jamais.

7 Dans l'œuvre graphique de Riquer, il y a une troisième apparition de ce motif féminin. Mais auparavant, il me semble qu'il faudrait s'interroger sur l'impact qu'aurait pu avoir sur cette troisième occurrence de ce motif, une affiche du grand artiste belge PrivatLivemont de 1896 pour l'Absinthe Robette. On y retrouve le motif du personnage féminin 
dénudé, de profil, seulement revêtu de voiles transparents, la tête légèrement en arrière, et qui tient au bout de ses bras levés un beau verre à pied qui contient l'absinthe dont l'affiche fait la propagande. Malgré l'aspect de publicité commerciale, il y a un caractère onirique qui se dégage de cette image au chromatisme pastel très harmonieux, à base de verts et de jaunes, sur lesquels se détachent la chevelure rousse ondulée, très Art Nouveau, de la femme et les lignes de contour de sa silhouette. Le fond est décoré d'un décor végétal, qui semble dégager des arômes prenants et obsédants qui peuvent faire référence aux effets pervers de la liqueur d'absinthe, la célèbre fée verte qui rendait fou, disait-on alors. Alexandre de Riquer connaissait bien l'œuvre de Privat-Livemont qu'il admirait et dont il reprend souvent la délicatesse du trait et l'harmonie de la gamme chromatique. On peut donc se demander si cette affiche de l'Absinthe Robette pourrait avoir eu une certaine influence sur l'affiche de son recueil de poésie publié en 1902 Anyor anses, mais ici, au contraire de ses premiers emprunts à l'art préraphaélite analysés au début, il va spiritualiser une image publicitaire.

La mort de l'épouse de Riquer, Lolita, en 1899, transforma sa vie et son inspiration poétique. Anyorances (Nostalgies), comme le titre l'indique, évoque la présence de l'amour perdu dans des poèmes où la nostalgie et la tristesse se mêlent à l'espoir d'une réunion avec la femme aimée dans un amour transcendant, « post mortem ». Riquer s'inspire de la poésie de Dante Gabriel Rossetti, un « Dante moderne et lumineux », comme il le qualifie et particulièrement de The Blessed Damozel (Rossetti, 1891). Le thème principal du livre apparaît synthétisé par l'affiche qui annonce le livre et qui montre ce désir de réunion avec l'amour perdu glosé dans les poèmes. La figure féminine symbolise les états d'âme du peintre-poète, à la fois sa profonde mélancolie, son désir de s'élever vers le ciel où se trouve l'aimée et son impossibilité à réaliser ce vœu. Le cadre de la scène est le bois et la montagne, c'est-à-dire le temple de la vie et le paradis terrestre. L'être féminin idéalisé (il a une couronne d'étoiles autour de la tête, signe de divinité équivalent à l'auréole employée par Rossetti pour sa Blessed Damozel) est situé au milieu d'une prairie couverte d'angéliques Il reprend la silhouette habituelle, corps vu de profil, couvert de longs voiles transparents, tête renversée en arrière, longue chevelure rousse bouclée, et il élève au bout de ses bras levés une coupe qui semble pleurer, exprimant ainsi un sentiment de tristesse, mais cette coupe représente aussi un idéal inaccessible, le « graal » du poète. Car cette matérialisation de l'âme du poète est enchainée à la terre, symbolisée par une grande pierre, elle ne peut se détacher de la matérialité de son corps, de là son désespoir. L'affiche est une visualisation des sentiments exprimés par le «je poétique » dans le poème XXVI (p. 34-35), dont je vous traduis le fragment suivant :

Je passais en chantant sur terre

Jusqu'à perdre la voix,

Et la prairie, la forêt et la montagne

Etaient en fleurs...

Alors que je chantais avec plus de force et de vitalité

Le malheur m'a frappé !...

Il m'a laissé abattu, sans guide, Regardant l'au-delà

Là où s'est perdu mon amour qui fleurissait, Là où va mon cœur.

Depuis la terre, je ne peux y arriver

À cette étoile brillante ;

Je me traîne et je ne peux pas y aller

Si loin, jusqu'au ciel!

Asservi par de lourdes chaînes

Comment puis-je voler? 
pour un ex-libris dun mysterieux possesseur, dont nous n'avons que les initiales AL, qui pour un ex-libris d'un mystérieux possesseur, dont nous n'avons que les initiales AL, qui sont celles d'Augusta Laborde, la sœur de la deuxième épouse de Riquer, mais qui pourraient aussi se lire «A Lolita ", une forme dissimulée de faire allusion au grand amour de l'artiste.

Une variante de cette composition ascensionnelle verticale se trouve dans une autre illustration de Crisantemes, celle de la p. 65 qui, elle aussi, avait servi de couverture au numéro 10, de décembre 1898 de la revue Luz, et qui était reproduite en plus grand format. Dans le livre, cette image ouvre un poème en prose, entièrement sous la forme interrogative, où la voix poétique s'adresse à la nuit afin qu'elle lui dévoile tous ses mystères, nuit espace de la rêverie, de l'amour, de la résurrection sous l'influence bénéfique de la lune. Une des questions du texte nous intéresse particulièrement parce qu'elle semble une glose de la vignette aperturale : « Les vierges mélancoliques, pourquoi cherchent-elles dans la lointaine étoile qui brille dans l'obscurité le point d'union entre deux âmes? »

11 Nous avons ici une composition horizontale très allongée, où nous retrouvons la même fusion entre le personnage féminin et la nature que dans l'autre vignette étudiée de Crisan temes, personnage qui semble une émanation de la nature et qui se trouve au bord d'un étang, ce qui fait que nous ne savons pas s'il s'agit d'une nymphe ou d'une naïade, mais peu importe puisqu'au fond, nous savons par le texte qu'elle est une visualisation du sentiment d'union amoureuse idéale qui germe dans la nuit et qui doit réunir l'âme de la femme et la lointaine étoile, l'hypothétique et mystérieuse âme sœur qui brille dans la nuit. Le motif iconographique féminin a changé, la position n'est plus la même, même si le buste en diagonale de la jeune fille semble s'élever légèrement, son mouvement se dirige surtout vers la gauche de la composition, son regard comme aimanté, est fixé sur la lointaine étoile à l'extrémité gauche alors qu'elle est décalée, elle, à droite pour ouvrir cet espace rempli par la flore de la nature et le ciel. Le croissant de lune, qui sert de contrepoint à l'étoile, est placé derrière elle, il lui est donc associé, et l'on sait que la lune est à la fois symbole de féminité et d'imagination, de rêve. La jeune vierge, avec les mains réunies sous son menton, semble prier, émettre un vœu ardent, alors que sa chevelure abondante, presque aquatique, est semée de marguerites, symbole d'innocence et d'amour.

12 Pour en revenir donc à notre problématique, l'étude des rapports entre imagination et mémoire dans la production de l'image, je pense qu'il s'agit d'un phénomène proche de l'intertextualité en littérature. À l'origine, et dans la mémoire que j'appellerai plutôt les sources iconographiques de l'image, il y aurait la symbolique traditionnelle et ancienne du corps féminin que l'on trouverait chez Riquer dans les allégories de l'Art et de l'Industrie illustrant l'affiche pour la III Exposition des Beaux-Arts et Industries Artistiques de Barcelone de 1896. Et on voit bien que, comme dans l'intertextualité, c'est la différence de contextualisation, de cadre, qui va modifier le sens de la figure féminine de George Frampton d'une Sainte Cécile en une allégorie de l'art chez Riquer, ici essentiellement par l'utilisation des attributs, des objets iconographiques. Pour les exemples suivants, les figures féminines ascensionnelles étudiées, le processus va devenir plus complexe, sauf pour l'hommage à Cervantes de la couverture de La Ilustración Artístic $a$ où la figure féminine, même si elle a toute la sensualité de l'Art Nouveau, n'est qu'une allégorie de la gloire littéraire. Même si la peinture de 1887 est essentiellement décorative, le personnage féminin est toutefois connoté par son attitude, ce 
renversement érotique du corps, et par les attributs, ces pavots qu'il sent et qui semblent le transporter, grâce à l'opium qui en dérive, dans des paradis artificiels. Dans l'occurrence suivante du même motif, celle de la p. 20 de Crisantemes, on passe subtilement de l'érotisme à une sorte de mysticisme, qui n'est pas exempt, et c'est bien connu, de sensualité, à la fois un désir de fusion panthéiste avec la nature et une sorte de visualisation du désir d'au-delà de l'âme de l'artiste, qui nous sont explicités et révélés par le texte du poème. On pourrait dire la même chose de l'illustration de la p. 65 de Cris antemes ainsi que de l'affiche du recueil poétique Anyoranses, où le texte dévoile le contenu symbolique de l'image et en même temps la décrit. Il est évident qu'il y a une complémentarité totale entre texte et image dans l'œuvre de Riquer. Je me suis longtemps demandé comment se réalisait chez lui le processus de création, et j'ai émis l'hypothèse de ce que j'ai appelé une cosmovision, c'est-à-dire que je pense qu'une fois qu'il avait déterminé le type féminin qui convenait à ses états d'âme, à ses sentiments, il devait le mettre en scène à la fois par une attitude et un décor qui, je pense, lui sont personnels ou tout au moins sont une intériorisation et une sélection du monde naturel, et qu'il en avait donc une vision dans son imagination. À la fin de sa vie, Riquer regrettait d'ailleurs de n'avoir pas pu réaliser matériellement tous les chefs-d'œuvre qu'il avait imaginés dans son esprit. Et si l'on peut penser que l'œuvre est créée par l'émotion, le sentiment de l'artiste, et donc certainement un début d'écriture du poème, une autre partie plus descriptive du texte semble décrire l'image conçue dans l'esprit de l'artiste. Au final, texte et image se mêlent de façon inextricable dans un univers riquerien que je considère personnel et original à la fois par le traitement de la figure féminine, disposée dans des formats très verticaux, de véritables makimonos, qui lui permettent de déployer des compositions ascensionnelles savantes et raffinées, dans lesquelles des nymphes vêtues de voiles légers semblent vouloir s'élever vers le ciel, source d'une lumière mystique dont les rayons s'enfoncent dans leurs yeux extasiés, reflets de leur âme, et par la douce et ingénue poésie des fonds des compositions - forêts, prairies, étangs -, une poésie qui s'exprime par le vague des fonds un peu flous, les nuances de plans gris, légers, voiles mystérieux qui nous ouvrent les voies de l'imagination et du rêve, qui se perdent également, d'autre part, lorsque l'on veut déchiffrer l'insondable mystère, l'ésotérique beauté contenue dans une simple tige, une corolle, auxquelles Riquer donne, en les stylisant, la perfection idéale de l'arabesque, cette courbe elliptique qui pour les esthètes de l'époque, était la quintessence de l'art.

C'est donc cette mise en scène du corps féminin évanescente, cette transcendance du motif, cet aspect de rêve vers un au-delà de ses compositions ascensionnelles qui permettent, à mon avis, d'inclure Alexandre de Riquer dans la liste, non pas des imitateurs et copistes d'un style et d'une mode, sans qu'il y ait assimilation et intériorisation du modèle, ce qui aurait pu être le cas par exemple de la femme sensuelle Art Nouveau de l'Absinthe Robette de Privat-Livemont pour l'affiche d'Anyoranses si nous ne connaissions pas le processus de spiritualisation de l'image opéré par Riquer, mais dans celle des authentiques artistes visionnaires du Symbolisme. 


\section{NOTES}

1. CORNUDELLA i CARRÉ, Rafael, «Sobre els cartells d'Alexandre de Riquer i les seves fonts », Loc us Amoenus, Barcelona, núm. 1, 1995, p. 233.

\section{RÉSUMÉS}

Me propongo reflexionar sobre la utilización a finales del siglo XIX de la alegoría femenina como vector de espiritualización, como imagen del alma, tomando el ejemplo del poeta, dibujante y gra fista modernista Alexandre de Riquer. ¿Es posible para el creador interiorizar y modernizar, es de cir adaptar a la estética simbolista de la época, la iconografía tradicional y recurrente del cuerpo femenino alegórico? El problema que me planteo es en definitiva intentar ver si se trata, a pesar de la utilización de modelos femeninos anteriores, de una inspiración mediatizada, o si la nueva i magen femenina simbolista tiene, a pesar de todo, algo de original, de recreación, y para eso en el caso de Riquer, el paralelismo entre texto poético e imagen ayuda mucho a elucidar la cuestión.

\section{INDEX}

Palabras claves : Riquer (Alexandre), allégorie, symbolisme, modernisme catalan

\section{AUTEUR}

\section{ELISEO TRENC}

Université de Reims 